\title{
Plunging cavities
}

\section{CLANET}

LadHyX, UMR7646 du CNRS, Ecole Polytechnique, 91128 Palaiseau, France
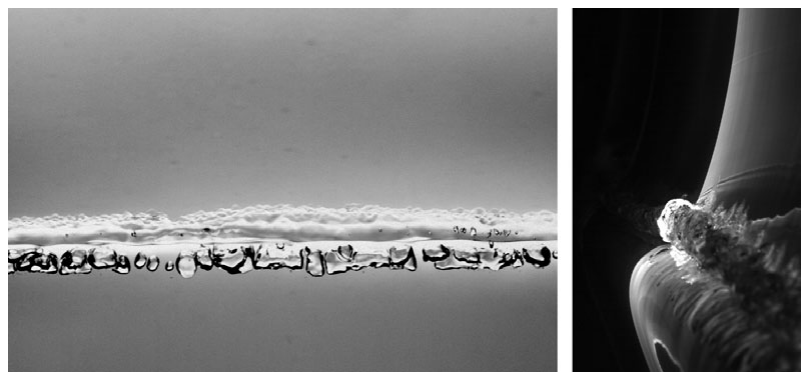

When a wave breaks, the tip forms a liquid sheet which impinges the base and creates an air cavity which breaks into bubbles. Gomez-Ledesma, Kiger \& Duncan (J. Fluid Mech., this issue, vol. 680, 2011, pp. 5-30) have conducted a nice experiment on this problem, enabling them to discuss both the inclination of the jet and the effect of its translation. This work has interesting links with other transient cavities.

Key words: bubble dynamics, free-surface flows, jets, wave breaking

\section{Introduction}

Water waves break in a wide range of conditions (Battjes 1988; Banner \& Peregrine 1993). In recent years, surf-zone breaking waves (Kimmoun \& Branger 2007) and bow waves (Shakeri, Tavakolinejad \& Duncan 2009; Noblesse et al. 2008) have received considerable attention due to their connection to coastal and naval applications. Mathematically, this breaking is an example of singularity formation: the equations are derived in the small slope limit but their solution develops beyond the vertical (Pomeau et al. 2008a,b). Physically, breaking waves play a major role in energy dissipation (Jessup et al. 1997) and air entrainment across the interface (Deane \& Stokes 2002). Examples of breaking waves are shown in figure 1, and in top and sideview in the figure by the title. We observe in figure 1(a) that beyond the vertical, the tip of the wave plunges towards the front. The jet impact is visualised in figure $1(b)$. The article by Gomez-Ledesma et al. (2011) is dedicated to a precise experimental and theoretical study of the resulting cavity. We discuss here the link between their nice study and other types of transient cavities.

\section{Overview}

Three examples of cavities are presented in figure 2. In the first sequence (figure $2 a$ ), extracted from Zhu, Oguz \& Prosperetti (2000), a vertical cylindrical water jet of diameter $D_{j}=5.4 \mathrm{~mm}$ and velocity $U_{0}=1.65 \mathrm{~m} \mathrm{~s}^{-1}$, initially connects to the pool without any air entrainment. Then, a bulge is generated on the liquid jet. The first image of the sequence presents the arrival of the bulge at the surface of the pool. Subsequent images show that the impact of the bulge creates a cavity. Zhu et al. (2000) have characterized this type of cavity and found that the crater size $D_{c}$ scales as $D_{c} / D_{j} \sim F r^{1 / 4}$, where $F r=U_{0}^{2} / g D_{j}$ is the Froude number. What we 
(a)

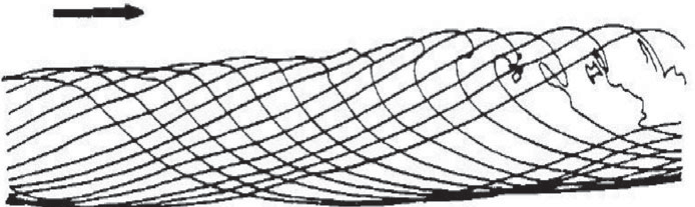

$0 \quad 10 \mathrm{~cm}$

FIGURE 1. (a) Observed crest deformation of a plunging breaking wave extracted from Bonmarin (1989). (b) Image of wave breaking from Shakeri et al. (2009).

(a)

(b)
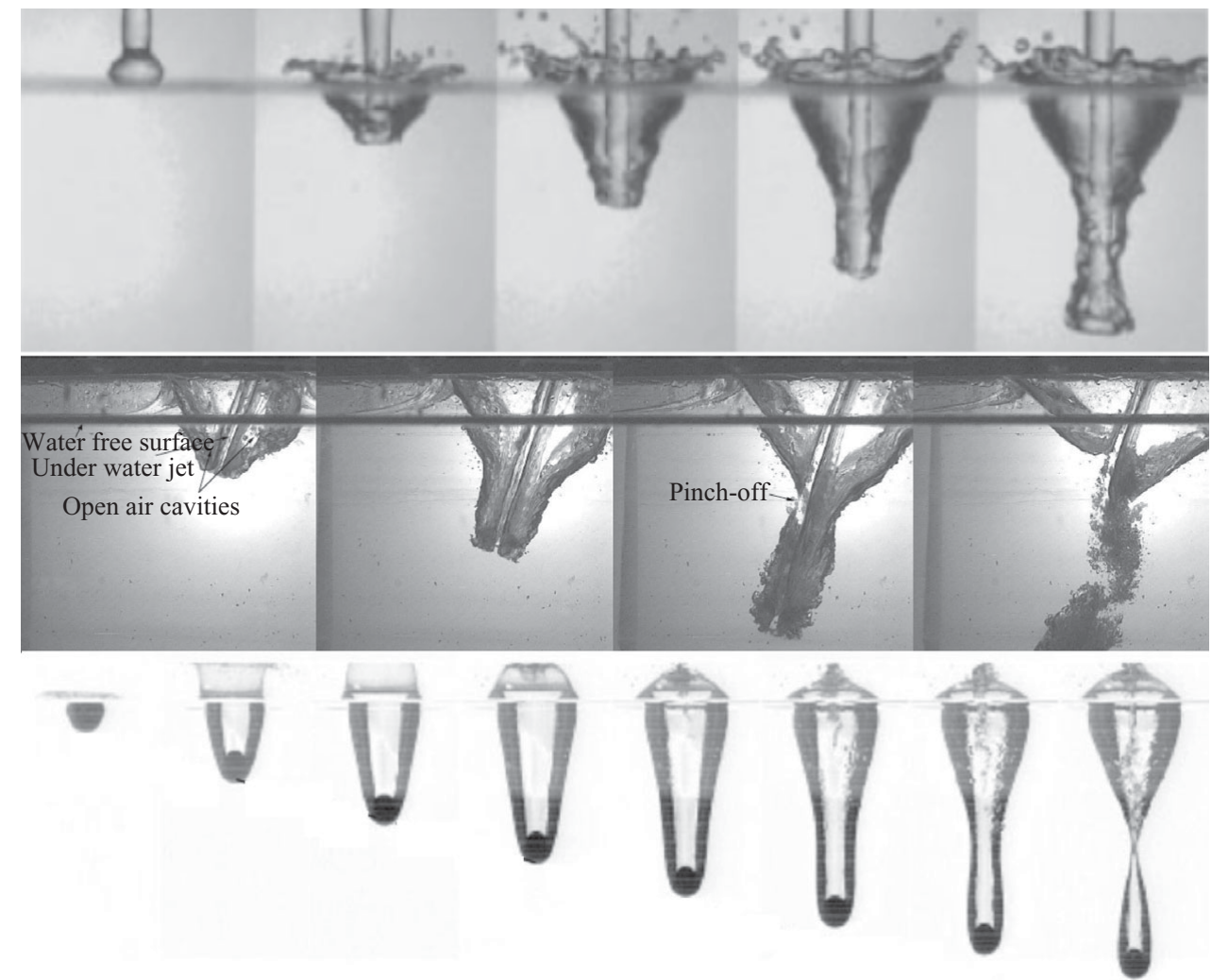

FIGURE 2. (a) Cavity created by a vertical cylindrical water jet of diameter $D_{j}=5.4 \mathrm{~mm}$ and velocity $U_{0}=1.65 \mathrm{~m} \mathrm{~s}^{-1}$ (from Zhu et al. 2000). (b) Cavity created by the impact of an inclined water sheet of thickness $T_{j}=2.9 \mathrm{~mm}$ and velocity $V_{j}=3.32 \mathrm{~m} \mathrm{~s}^{-1}$. The liquid sheet is translating from right to left at velocity $V_{t}=0.15 \mathrm{~m} \mathrm{~s}^{-1}$ (from Gomez-Ledesma et al. 2011). (c) Cavity created during the impact of a solid sphere of diameter $D_{0}=15.6 \mathrm{~mm}$ and velocity $U_{0}=3.7 \mathrm{~m} \mathrm{~s}^{-1}$ (from Duclaux et al. 2007). 
(a)

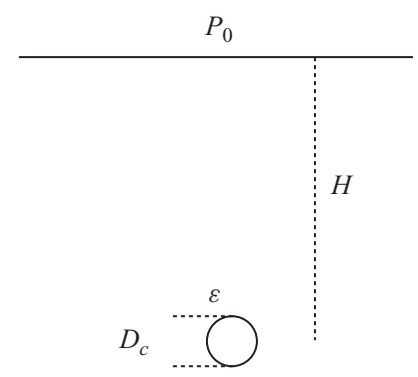

(b)

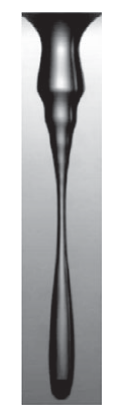

(c)

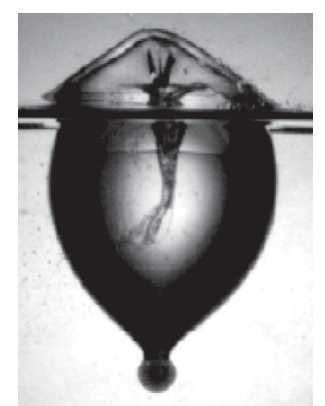

FiguRE 3. (a) Basic model developed to capture the crater size. (b) Impact of a steel sphere in water $\left(R_{0}=0.79 \mathrm{~mm}\right.$ and $\left.U_{0}=3.1 \mathrm{~m} \mathrm{~s}^{-1}\right)$. (c) Impact of a polypropylene sphere $\left(R_{0}=2.5 \mathrm{~mm}\right)$ in a viscous pool $(\eta=1 \mathrm{~Pa} \mathrm{~s})$ with velocity $U_{0}=28 \mathrm{~m} \mathrm{~s}^{-1}$.

retain here is that a bulge is needed to generate the cavity. Without the bulge, the jet connects directly to the pool. In the second sequence (figure $2 b$ ), we present a cavity as observed by Gomez-Ledesma et al. (2011). The cavity is created by an inclined liquid sheet of thickness $T_{j}=2.9 \mathrm{~mm}$ and velocity $V_{j}=3.32 \mathrm{~m} \mathrm{~s}^{-1}$. In this case, the liquid sheet is also translating from right to left with velocity $V_{t}=0.15 \mathrm{~m} \mathrm{~s}^{-1}$. The translation induces a lateral displacement of the order of $V_{t} \tau_{t}$ after the time $\tau_{t}$. This displacement becomes of the order of $T_{j}$ at the time $\tau_{t} \sim T_{j} / V_{t}$, of the order of $50 \mathrm{~ms}$. One does not expect to observe an effect of the translation before $\tau_{t}$. Indeed, in the first two images, the cavity is symmetrical with respect to the liquid sheet. The symmetry breaks in the third image and the pinch-off occurs on the 'front' side of the cavity. Even though the general shape of the cavity is close to that observed with cylindrical jets, Gomez-Ledesma et al. (2011) report and characterize the sensitivity of the shape of the cavity to both the tilt angle and the translation velocity. Finally, in the last sequence extracted from Duclaux et al. (2007) (figure 2c), we present the cavity created by the vertical impact of a solid sphere of diameter $D_{0}=15.6 \mathrm{~mm}$ and velocity $U_{0}=3.7 \mathrm{~m} \mathrm{~s}^{-1}$. There again, the cavity is wider at the interface and pinches off for some critical elongation. The authors have shown that the size of the crater also scales as $D_{c} / D_{0} \sim F r^{1 / 4}$. A nice discussion of this type of cavity can also be found in Bergmann et al. (2009).

A common feature of the above three cavities is that they are all created by a local input of energy: the impact of the bulge in figure 2(a), the impact of the tip of the liquid sheet in figure $2(b)$ and the impact of the solid sphere in figure $2(c)$. We use this observation to develop a small argument on the size of such plunging cavities. The model is presented in figure $3(a)$. The energy $\mathscr{E}$ is deposited below the interface at the depth $H$. The size of the crater $D_{c}$ which results from this 'explosion' results from the balance $\mathscr{E} \sim \rho g H D_{c}^{3}$. If the energy $\mathscr{E}$ is deposited by an impactor (liquid jet or solid) of velocity $U_{0}$ and size $D_{0}$, we evaluate $\mathscr{E} \sim \rho U_{0}^{2} D_{0}^{3}$. The cavity size can finally be found by taking the limit $D_{c} \sim H$. The energy balance leads to the scaling $D_{c} / D_{0} \sim\left(U_{0}^{2} / g D_{0}\right)^{1 / 4}$, which has been reported by Zhu et al. (2000) and Duclaux et al. (2007). A more elaborate model is developed by Gomez-Ledesma et al. (2011), which captures all the features of the experiment up to pinching. 


\section{Future}

In the case of solid spheres, Aristoff \& Bush (2009) have shown that some surprises occur with sizes smaller than the capillary length. In this limit, surface tension can no longer be neglected and the cavity shape undergoes spectacular changes, as shown in figure $3(b)$. When the viscosity of the pool is markedly increased, Le Goff, Quéré \& Clanet (2011) report a new type of cavity where the sphere stops on a time scale shorter than the cavity collapse. In this limit, the cavity is no longer elongated as shown in figure $3(c)$. Both small size jets and flows with viscous pools are possible future avenues of research for liquid sheet cavities. However, in the context of a bow wave and plunging jets, a more probable future research direction is the characterization of the bubble size which results from the cavity collapse and the depth to which these bubbles are entrained by the jet.

\section{References}

Aristoff, J. M. \& Bush, J. W. M. 2009 Water entry of small hydrophobic spheres. J. Fluid Mech. 619, 45-78.

Banner, M. L. \& Peregrine, D. H. 1993 Wave breaking in deep water. Annu. Rev. Fluid Mech. 25, 373-397.

Battues, J. A. 1988 Surf-zone dynamics. Annu. Rev. Fluid Mech. 20, 257-293.

Bergmann, R., van der Meer, D., Gekle, S., van der Bos, A. \& Lohse, D. 2009 Controlled impact of a disk on a water surface: cavity dynamics. J. Fluid Mech. 633, 381-409.

Bonmarin, P. 1989 Geometric properties of deep-water breaking waves. J. Fluid Mech. 209, 405-433.

Deane, G. B. \& Stokes, M. D. 2002 Scale dependence of bubble creation mechanisms in breaking waves. Nature 418, 839-844.

Duclaux, V., Caillé, F., Duez, C., Ybert, C., Bocquet, L. \& Clanet C. 2007 Dynamics of transient cavities. J. Fluid Mech. 591, 1-19.

Gomez-Ledesma, R., Kiger, K. T. \& Duncan, J. H. 2011 Air entrainment due to a two-dimensional translating plunging jet. J. Fluid Mech. 680, 5-30.

Jessup, A. T., ZAPPA, C. J., Loewen, M. R. \& Hesany, V. 1997 Infrared remote sensing of breaking waves. Nature 385, 52-55.

Kimmoun, O. \& BRANGeR, H. 2007 A particle image velocimetry investigation on laboratory surf-zone breaking waves over a sloping beach. J. Fluid Mech. 588, 353-397.

Le Goff, A., Quéré, D. \& Clanet, C. 2011 Viscous cavities. Phys. Fluids (submitted).

MilleR, R. L. 1957 Role of vortices in surf zone prediction, sedimentation and wave forces. In Beach and Nearshore Sedimentation (ed. R. A. Davis \& R. L. Ethington), pp. 92-114. Society of Economic Paleontologists and Mineralogists.

Noblesse, F., Delhommeau, G., Guilbaud, M., Hendrix, D. \& Yang, C. 2008 Simple analytical relations for ship bow waves. J. Fluid Mech. 600, 105-132.

Pomeau, Y., Jamin, T., Le Bars, M., Le Gal, P. \& Audoly, B. 2008 a Law of spreading of the crest of a breaking wave. Proc. R. Soc. Lond. A 464, 1851-1866.

Pomeau, Y., Le Berre, M., Guyenne, P. \& Grilli, S. 2008b Wave-breaking and generic singularities of nonlinear hyperbolic equations. Nonlinearity 21, T61-T79.

Shakeri, M., TAVAKolinejad, M. \& Duncan, J. H. 2009 An experimental investigation of divergent bow waves simulated by a two-dimensional plus temporal wave marker technique. J. Fluid Mech. 634, 217-243.

Zhu, Y., Oguz, H. N. \& Prosperetti, A. 2000 On the mechanism of air entrainment by liquid jets at a free surface. J. Fluid Mech. 404, 151-177. 